\title{
Efecto del amamantamiento restringido y complementación alimenticia sobre el peso y cortisol en becerros Simbrah
}

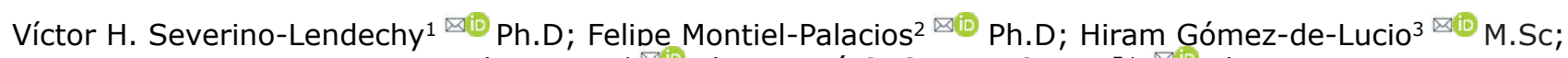
Jorge A. Peralta-Torres ${ }^{4}$ Ph.D; José C. Segura-Correa ${ }^{5 *} \bowtie(\mathbb{D}$ Ph.D.

1 Universidad Autónoma de Chiapas, Centro de Estudios Etnoagropecuarios. San Cristóbal de las Casas, Chiapas. México. 2Universidad Veracruzana, Facultad de Medicina Veterinaria y Zootecnia. Unidad Veracruzana, Veracruz, Veracruz. México. ${ }^{3}$ Tecnológico Nacional de México/Instituto Tecnológico de Conkal. División de Estudios de Posgrado e Investigación, Yucatán, México. ${ }^{4}$ Universidad Juárez Autónoma de Tabasco, División Académica de Ciencias Agropecuarias. Tabasco, México.

${ }^{5}$ Universidad Autónoma de Yucatán, Facultad de Medicina Veterinaria y Zootecnia. Mérida, Yucatán, México.

*Correspondencia: jose.segura52@hotmail.com

Recibido: Febrero 2020; Aceptado: Agosto 2020; Publicado: Noviembre 2020.

\section{RESUMEN}

Objetivo. Determinar el efecto del amamantamiento restringido, destete precoz y complementación alimenticia sobre las ganancias de peso y concentración plasmática de cortisol en becerros Simbrah. Materiales y métodos. Se utilizaron 120 becerros lactantes de 15 días de edad, distribuidos en dos tratamientos: T1) becerros con amamantamiento restringido, destete precoz y complementación alimenticia ( $n=30$ machos $/ n=30$ hembras) y T2) becerros con amamantamiento continuo sin complementación alimenticia $(n=30$ machos $/ n=30$ hembras). Los becerros se pesaron al inicio del experimento, y cada 15 días para evaluar los cambios en peso corporal del destete hasta los 10 meses de edad. Los muestreos sanguíneos se realizaron al inicio del estudio y durante 10 días, entre las 7:30 y 8:30 am. Las variables de respuesta se evaluaron usando modelos lineales generales. Resultados. El peso $(310.1$ y $268.5 \mathrm{~kg})$ y ganancia de peso postdestete $(0.980 \mathrm{~kg} / \mathrm{día}$ y 0.800 $\mathrm{kg} / \mathrm{día}$ ) fue mejor en el tratamiento T1 que, para los becerros en T2 (168.3 y $159.6 \mathrm{~kg} ;$ y -0.500 y - $0.480 \mathrm{~kg} /$ día) para machos y hembras, respectivamente. La concentración de cortisol (ng/mL) fue mayor en T1 comparado con T2 del día 0 al día $3(p<0.05)$, pero el día 4 no se encontró diferencia. El T1 permitió el destete a los 3.5 meses de vida de los becerros e incrementó la ganancia de peso posdestete. Conclusiones. El peso vivo y ganancia de peso posdestete fue mejor en los animales del tratamiento T1; sin embargo, los niveles de cortisol plasmáticos se incrementaron al inicio del estudio para disminuir posteriormente.

Palabras clave: Destete precoz; nutrición; estrés; amamantamiento; bienestar animal (Fuente CAB).

\section{ABSTRACT}

Objective. Determine the effect of restricted suckling, early weaning and feed supplementation on weight gains and plasma cortisol concentration of Simbrah calves. Materials and methods. One hundred and twenty 15-day-old lactating calves were distributed in two treatments: T1) calves with restricted suckling, early weaning and feed supplementation ( $n=30$ males $/ n=30$ females); and T2)

Como citar (Vancouver).

Severino-Lendechy VH, Montiel-Palacios F, Gómez-de-Lucio H, Peralta-Torres JA, Segura-Correa JC. Efecto del amamantamiento restringido y complementación alimenticia sobre el peso y cortisol en becerros Simbrah. Rev MVZ Córdoba. 2021; 26(1):e1958. https://doi.org/10.21897/rmvz.1958 
calves with continuous suckling without feed supplementation ( $n=30$ males $/ n=30$ females). Calves were weighed at the beginning of the experiment, and every 15 days to assess changes in body weight from weaning unto 0 months of age. Blood sampling was carried out at the start of study and for 10 days between 7:30 and 8:30 am. Response variables were evaluated using general linear models. Results. The weight ( 310.1 and $268.5 \mathrm{~kg})$ and post-weaning weight gain $(0.980$ and 0.800 $\mathrm{kg} /$ day) was better in the T1 treatment than, for calves in T2 (168.3 and $159.6 \mathrm{~kg}$; and -0.500 and $-0.480 \mathrm{~kg} /$ day) for males and females, respectively. The cortisol concentration $(\mathrm{ng} / \mathrm{mL})$ was higher in T1 compared to T2 from day 0 to day $3(p<0.05)$, but on day 4 no difference was found. T1 allowed weaning at 3.5 months of calf life and increased post-weaning weight gain. Conclusions. Live weight and post-weaning weight gain were better in T1 treatment animals; however, plasma cortisol levels were increased at the star of the study to subsequently decrease.

Keywords: Early weaning; nutrition; stress; suckling; animal welfare (Source CAB).

\section{INTRODUCCIÓN}

El amamantamiento controlado o restringido, así como el destete temporal, temprano o precoz son técnicas de crianza aplicadas en los bovinos de carne $(1,2)$, que buscan criar de manera parcial, temporal o total al becerro después del nacimiento. El uso de complementos alimenticios (sustituto de leche, alimento balanceado y forraje verde o seco), y el amamantamiento restringido tiene como finalidad: 1 ) disminuir de manera gradual la dependencia de la cría a la leche materna y desarrollar la capacidad ruminal, lo más pronto posible, para ser destetada a corta edad. 2) mejorar el comportamiento reproductivo (anestro posparto) de la vaca al disminuir el estrés de la lactancia y el vínculo vaca-becerro que se genera por el amamantamiento $(3,4,5)$.

Se han notificado mejoras en algunas características reproductivas de las vacas (reinicio temprano de la ciclicidad, mejora en la condición corporal y aumento en la tasa de gestación) $(6,7)$. Sin embargo, los becerros, al tener un mayor consumo de leche materna y bajo o nulo consumo de alimentos sólidos (alimento balanceado y/o forrajes), no tienen un desarrollo ruminal eficiente. Por lo tanto, al momento del destete, las crías sufren cambios bruscos a nivel físico, social y fisiológico (aumento de bramidos, agitación, caminar constantemente y reducción del consumo alimenticio) que pueden afectar su estatus quo generando estrés en los animales $y$ en algunos casos la muerte $(5,8,9)$. Bajo estas circunstancias, el uso de los métodos de amamantamiento restringido o destete precoz controlado son positivos para las vacas $(2,10)$; pero negativos para los becerros, por la falta de bienestar animal, al causar estrés en ellos al separarlos de la madre (1).
El uso de estas tecnologías en las regiones tropicales es limitado $(11,12,13)$, debido a varios factores que desincentivan en los productores su implementación, como los costos del alimento y el manejo constante de los animales. Por lo tanto, el manejo que reciba el becerro durante el proceso de separación de la madre a edad temprana, debe ser empleado de manera estratégica. Esto es, las crías deben ser complementadas con alimento acorde a su etapa fisiológica, facilitando su adaptación al cambio de dieta (leche-alimento sólido). Al mismo tiempo, se deben utilizar indicadores de bienestar animal, que permitan conocer el nivel de estrés que las crías experimentan durante el crecimiento, para reducir el estrés y hacerla más productiva $(10,14)$.

Pocos estudios han determinado el efecto de la complementación alimenticia y nivel de estrés, causados por el destete, en el comportamiento posdestete en ganado de carne, especialmente en ganado Simbrah, en las regiones tropicales $(5,10,11,13)$. El objetivo de este estudio fue determinar el efecto del amamantamiento restringido, destete precoz y la complementación alimenticia sobre la ganancia de peso y concentración sérica de cortisol en becerros Simbrah al destete y posdestete.

\section{MATERIALES Y MÉTODOS}

Los procedimientos realizados durante este experimento se apegaron a la Norma Oficial Mexicana (15), publicada en el Diario Oficial de la Federación sobre las Especificaciones técnicas para la producción, cuidado y uso de los animales de laboratorio y experimentales. 
Ubicación geográfica de la unidad de producción. El estudio se realizó en una unidad de producción pecuaria dedica a la producción de becerros para abasto. El rancho se localizada en Macuspana, Tabasco, México $\left(17^{\circ} 45^{\prime} 17^{\prime \prime} \mathrm{N}\right.$ y $92^{\circ}$ $\left.33^{\prime} 32^{\prime \prime} \mathrm{W}\right)$, a una altura de $10 \mathrm{msnm}$, con clima tropical, temperatura y precipitación media anual de $26.4^{\circ} \mathrm{C}$ y $3186 \mathrm{~mm}$, respectivamente (16).

Características del estudio y de los becerros. Previo al experimento, se realizó un empadre de 90 días en 300 vacas con $75 \pm 15$ días posparto y $3.5 \pm 1.5$ partos. Las vacas fueron sincronizadas con progesterona natural y apareadas con sementales previamente evaluados andrológicamente, para dar servicio efectivo. El diagnóstico de gestación se realizó a los 60 días, al final del empadre. Posterior al parto, se seleccionaron 120 becerros $(n=60$ machos y $n=60$ hembras) Simbrah lactantes $(15 \pm 2$ días de nacidos y $63.8 \pm 6.5 \mathrm{~kg}$ de peso vivo), e identificados conforme a los registros existentes de la unidad de producción.

La desparasitación interna de los becerros se realizó con Clorhidrato de Levamisol al $15 \%$ (Lab. Genfar, dosis $1 \mathrm{~mL} / 20 \mathrm{~kg}$ de peso vía intramuscular (im)) y la desparasitación externamente con Flumetrina al 1\% (Lab. Bayer, dosis $10 \mathrm{~mL} / 100 \mathrm{~kg}$ de peso vía tópica). En adición, los animales recibieron un complejo hidróxido férrico hierro (Lab. Bayer, dosis 1000 mg vía im) y vitaminas liposolubles como Vitamina A 500.000 UI, Vitamina D3 75.000 UI, Vitamina E 50 UI (Lab. Bayer, dosis $2 \mathrm{~mL}$ vía im). Los animales se vacunaron a los 3 y 9 meses de vida contra el virus de rabia usando una vacuna inactivada (cepa Pasteur RIV: $\geq 2$ U.I; Lab. Intervet, dosis $2 \mathrm{~mL}$ vía im) y contra Bacillus anthracis (cepa Sterne con una concentración mínima de 2,000,000 de esporas no encapsuladas por $\mathrm{mL}$; Lab. Bayer, dosis 1 $\mathrm{mL}$ vía subcutánea). Así mismo, los animales se vacunaron contra Pasteurella multocida tipo A y D, Mannheimia (Pasteurella) haemolytica A-1, Clostridium chauvoei y Clostridium septicum, (Lab. MSD, dosis $5 \mathrm{~mL}$ vía im).

Tratamientos. El experimento duró 303 días (10 meses), los becerros se identificaron con números progresivos y se distribuyeron al azar a los siguientes tratamientos. T1) 60 becerros con amamantamiento restringido y complementación alimenticia durante destete y posdestete $(n=30$ machos $/ n=30$ hembras), y T2) 60 becerros con amamantamiento continuo sin complementación alimenticia durante destete y posdestete $(n=30$ machos $/ n=30$ hembras). Los becerros del tratamiento T1 fueron separados de la madre a los 15 días de nacidos y se encerraron en un corral con piso firme, semi-techado y con espacio vital de $5 \mathrm{~m}^{2} /$ animal. Los becerros se amamantaron una vez al día por la mañana (7:00 a 8:00 horas) hasta el destete (3.5 meses de edad) trayendo a las madres al corral. En adición, los becerros recibieron complementación alimenticia con alimento balanceado ad libitum ( $21 \%$ de PC y $75.26 \%$ de TND). Posterior al destete, la complementación alimenticia se dio a razón de $2.5 \%$ de peso vivo por animal/ día. Durante todo el proceso se ofreció forraje seco, dispuesto en pacas de pasto Humidicola (Brachiaria humidicola) y agua ad libitum.

Los becerros del T2 permanecieron con sus madres desde el nacimiento, las cuales estuvieron en un sistema de pastoreo rotacional (cada 5 días/ potrero) en 30 potreros de 5 ha. Los potreros estaban sembrados con pasto MG5 (Brachiaria brizanta; $10 \%$ de PC) y pasto Humidicola ( $7.5 \%$ de $P C)$, en un área de 150 ha con disponibilidad a agua potable en bebederos móviles y sales minerales a libre acceso ( $8 \%$ de fósforo). Posteriormente, al alcanzar los 7 meses de edad los becerros fueron destetados y manejados bajo el mismo sistema de alimentación mencionado anteriormente.

Medición del cambio de peso corporal. Al inicio del estudio (15 días de edad), los becerros de ambos tratamientos ( $\mathrm{T} 1$ y $\mathrm{T} 2$ ) se pesaron con una báscula ganadera con capacidad para 2000 kg (Revuelta ${ }^{\circledR}$, México), y posteriormente cada 15 días hasta los 303 días de edad. Con la información obtenida se determinó el peso al destete, ganancia de peso al destete, ganancia de peso total al destete, peso posdestete, ganancia de peso posdestete, ganancia de peso total posdestete y ganancia de peso total.

Toma de muestras sanguíneas y determinación sérica de cortisol. Los muestreos sanguíneos se realizaron entre las 7:30 y 8:30 am, con la finalidad de determinar la concentración sérica de cortisol, debido al efecto de la separación del becerro de la vaca. Los becerros de los tratamientos T1 y T2 se muestrearon del día 0 (inicio tratamiento) al día 10 (30 min antes y 30 min después de separados de la madre), mediante la punción de la vena coccígea, con aguja calibre $21 \mathrm{G}$ x $38 \mathrm{~mm}$ y tubos Vacutainer ${ }^{\circledR}$ de $6 \mathrm{~mL}$, sin anticoagulante. 
Las muestras se centrifugaron a $700 \mathrm{~g} / 10$ min, utilizando una centrifuga marca HETTICH Zentrifugen ${ }^{\circledR}$ (modelo D-78532 Tuttlingen, Berlín, Alemania), en un tiempo no mayor a 4 horas después de tomadas las muestras. El suero se separó y se hicieron alícuotas que se congelaron a $-20^{\circ} \mathrm{C}$ hasta la determinación de la concentración de cortisol $(\mathrm{ng} / \mathrm{mL})$, mediante enzimoinmunoensayo (ELISA) en fase sólida, en el Laboratorio de Fisiología de la Facultad de Medicina Veterinaria y Zootecnia de la Universidad Veracruzana.

Se utilizaron kits comerciales de cortisol (Cortisol, EIA-1887, DRG Diagnostics, Alemania), siguiendo las indicaciones del fabricante, donde la especificidad de anticuerpos para reacción cruzada de cortisol y corticosterona era de 100 y $45 \%$, respectivamente, y la precisión de la variación intra e inter-ensayo era de $5.63 \%$ y $6.93 \%$, respectivamente. La lectura de las placas de ELISA se hizo a 450 nanómetros en un lector de ELISA marca HLAB ${ }^{\circledR}$ (modelo HReader1, HLab supply LTD, Berlín, Alemania).

Análisis estadístico. Los pesos al inicio del estudio al destete, posdestete, ganancia de peso total del destete hasta los 303 días y ganancia de peso total del inicio del estudio a los 303 días, se analizaron utilizando un modelo general que incluyó los efectos fijos de tratamiento y sexo del becerro. Las variables ganancia diaria del inicio del estudio hasta los 303 días y del destete a los 303 días, así como los datos de la concentración sérica de cortisol se evaluaron mediante un modelo lineal general donde el animal se consideró la unidad experimental y los pesajes de los animales o los muestreos de cortisol, las medidas repetidas.
El modelo general que describió las variables con medidas repetidas se presenta a continuación:

$Y_{i j k l}=m+t_{i}+s_{j}+a\left(t^{*} s\right)_{i j k}+r_{1}+e_{i j k l}$

Donde:

$\mathrm{Y}_{\mathrm{ijkl}}=$ cualquiera de las variables de respuesta con medidas repetidas.

$\mathrm{m}=$ la media general de los datos de la variable de interés.

$t_{i}=$ efecto fijo del i-ésimo tratamiento.

$\mathrm{s}_{\mathrm{j}}=$ efecto fijo del j-ésimo sexo del becerro.

$\mathrm{a}\left(\mathrm{t}^{*} \mathrm{~s}\right)_{\mathrm{ijk}}=$ efecto aleatorio del k-ésimo animal anidado dentro de las combinaciones tratamientosexo (error experimental, NID $\left(0, \sigma^{2}\right)$ ).

$r_{1}=$ efecto fijo de la l-ésima medición.

$\mathrm{e}_{\mathrm{ijkl}}=$ efecto aleatorio del error residual $\operatorname{NID}\left(0, \sigma^{2}\right)$.

Las medias de tratamiento y sexo se compararon utilizando valores de $\mathrm{p}$ ajustados para múltiples comparaciones por pruebas de rango de Tukey. Todos los análisis estadísticos se realizaron utilizando el paquete SPSS (17).

\section{RESULTADOS}

Se encontró diferencia entre tratamientos para las características pre y posdestete, así como de sexo y número de pesaje o muestreo. El peso al destete de los becerros machos y hembras, la ganancia diaria de peso y ganancia de peso total al destete para los animales del tratamiento T1 fue menor $(p<0.05)$ con respecto al tratamiento T2. Sin embargo, posterior al destete, los becerros (machos y hembras) del tratamiento T1 tuvieron mayor peso posdestete, ganancia diaria de peso y ganancia de peso total con respecto a T2 (Tabla 1, Figura 1).

Tabla 1. Medias \pm desviación estándar por tratamiento y sexo para características productivas pre y posdetete en becerros Simbrah en el trópico de México.

\begin{tabular}{|c|c|c|c|c|}
\hline \multirow{2}{*}{$\begin{array}{c}\text { Tratamiento } \\
\text { Sexo/Variable }\end{array}$} & \multicolumn{2}{|c|}{ T1 } & \multicolumn{2}{|r|}{ T2 } \\
\hline & Macho $(n=30)$ & Hembra $(n=30)$ & Macho $(n=30)$ & Hembra $(n=30)$ \\
\hline Peso inicial ( $\mathrm{kg} ; 15$ días de edad) & $63.5 \pm 7.0^{a}$ & $61.0 \pm 5.0^{\mathrm{a}}$ & $62.1 \pm 6.1^{a}$ & $60.1 \pm 5.5^{a}$ \\
\hline Peso al destete $(\mathrm{kg})^{*}$ & $104.3 \pm 6.4^{a}$ & $98.5 \pm 5.4^{a}$ & $213.3 \pm 35.3^{b}$ & $202.9 \pm 25.1^{c}$ \\
\hline Ganancia diaria de peso al destete (kg/día) & $0.450 \pm 0.2^{\mathrm{a}}$ & $0.400 \pm 0.5^{b}$ & $0.720 \pm 0.6^{c}$ & $0.680 \pm 0.7 c$ \\
\hline Ganancia de peso total al destete $(\mathrm{kg})$ & $40.5 \pm 5.2^{\mathrm{a}}$ & $36.5 \pm 6.2^{\mathrm{a}}$ & $151.2 \pm 30.7^{b}$ & $142.8 \pm 21.7^{b}$ \\
\hline Peso posdestete a 303 días $(\mathrm{kg})$ & $310.1 \pm 20.1^{a}$ & $268.5 \pm 21.5^{b}$ & $168.3 \pm 25.1^{c}$ & $159.6 \pm 24.2^{c}$ \\
\hline Ganancia diaria de peso posdestete (kg/día) & $0.980 \pm 0.3^{a}$ & $0.800 \pm 0.1^{\mathrm{b}}$ & $-0.500 \pm 0.2^{c}$ & $-0.480 \pm 0.3^{c}$ \\
\hline Ganancia de peso total posdestete $(\mathrm{kg})$ & $205.8 \pm 19.3^{a}$ & $168.9 \pm 19.3^{b}$ & $-45.0 \pm 7.1^{c}$ & $-43.3 \pm 7.1^{c}$ \\
\hline Ganancia de peso total $(\mathrm{kg})^{* *}$ & $246.3 \pm 12.2^{\mathrm{a}}$ & $205.4 \pm 13.2^{b}$ & $106.3 \pm 14.6^{c}$ & $99.5 \pm 14.6^{c}$ \\
\hline
\end{tabular}

$a, b, c$ Diferentes literales entre filas indican diferencia estadística $(p<0.05$; prueba de Tukey); T1= Amamantamiento restringido, destete precoz y complemento alimenticio; T2= Amamantamiento continuo sin complemento alimenticio. * Los becerros del T1 se destetaron a los 105 y los del T2 a los 210 días; ** ganancia de peso total de los 15 a los 303 días de edad. 


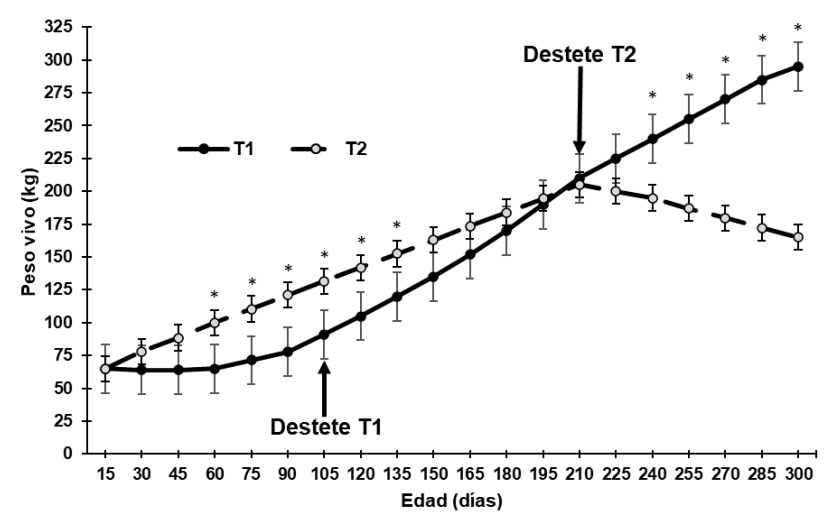

Figura 1. Peso vivo de becerros Simbrah con amamantamiento restringido, destete precoz y complemento alimenticio (T1) y amamantamiento continuo sin complemento alimenticio (T2) bajo condiciones tropicales.
No se encontró diferencia entre machos y hembras para la concentración de cortisol, pero sí de tratamiento y número de muestra. La concentración de cortisol $(\mathrm{ng} / \mathrm{mL}$ ) promedio en la primera muestra de sangre de los días 0 a 10 (antes de la separación del becerro de la madre) no mostraron diferencia estadística significativa entre los becerros del tratamiento T1 con respecto a T2. Sin embargo, en los muestreos de la segunda toma del día 0 a 3 , si se encontró diferencia $(p<0.05)$ entre tratamientos (Tabla 2$)$. Es decir, la concentración de cortisol plasmático en los becerros machos y hembras del T1 y T2 fue similar antes de la separación (día 0 a 10), pero no después de ella (día 0 a 3 ). A partir del día 4 la concentración fue similar antes y después de la separación de la cría de la madre (Tabla 2).

Tabla 2. Media \pm desviación estándar por tratamiento, sexo y número de muestra ( 30 min antes y 30 min después de la separación de la madre) para la concentración de cortisol de becerros Simbrah.

\begin{tabular}{|c|c|c|c|c|c|c|c|c|}
\hline \multirow{3}{*}{$\begin{array}{c}\text { Tratamiento } \\
\text { Sexo/Día }\end{array}$} & \multicolumn{4}{|c|}{ T1 } & \multicolumn{4}{|c|}{ T2 } \\
\hline & \multicolumn{2}{|c|}{$\operatorname{Machos}(n=30)$} & \multicolumn{2}{|c|}{ Hembras $(n=30)$} & \multicolumn{2}{|c|}{ Machos $(n=30)$} & \multicolumn{2}{|c|}{ Hembras $(n=30)$} \\
\hline & Muestra 1 & Muestra 2 & Muestra 1 & Muestra 2 & Muestra 1 & Muestra 2 & Muestra 1 & Muestra 2 \\
\hline 0 & $10.5 \pm 2.5^{a}$ & $56.2 \pm 8.0^{b}$ & $11.5 \pm 2.6^{\mathrm{a}}$ & $57.2 \pm 7.6^{b}$ & $9.9 \pm 2.7^{a}$ & $10.1 \pm 1.5^{\mathrm{a}}$ & $10.9 \pm 2.4^{a}$ & $11.7 \pm 1.7^{\mathrm{a}}$ \\
\hline 1 & $11.7 \pm 1.2^{\mathrm{a}}$ & $41.0 \pm 6.2^{b}$ & $11.5 \pm 1.2^{\mathrm{a}}$ & $40.0 \pm 7.1^{b}$ & $10.5 \pm 2.3^{a}$ & $11.5 \pm 2.1^{\mathrm{a}}$ & $10.1 \pm 2.3^{a}$ & $11.4 \pm 2.3^{a}$ \\
\hline 2 & $9.7 \pm 1.4^{\mathrm{a}}$ & $31.2 \pm 2.6^{b}$ & $9.9 \pm 1.5^{\mathrm{a}}$ & $30.2 \pm 2.6^{b}$ & $9.9 \pm 1.9^{a}$ & $10.5 \pm 1.0^{a}$ & $9.5 \pm 2.9^{a}$ & $10.4 \pm 1.0^{\mathrm{a}}$ \\
\hline 3 & $10.5 \pm 2.1^{\mathrm{a}}$ & $20.3 \pm 1.5^{b}$ & $10.3 \pm 2.2^{\mathrm{a}}$ & $21.3 \pm 1.7^{\mathrm{b}}$ & $10.9 \pm 1.8^{\mathrm{a}}$ & $10.5 \pm 2.9^{a}$ & $10.7 \pm 1.8^{a}$ & $10.8 \pm 2.5^{a}$ \\
\hline 4 & $9.7 \pm 1.5^{\mathrm{a}}$ & $10.1 \pm 2.7^{a}$ & $9.5 \pm 1.9^{a}$ & $10.2 \pm 2.5^{a}$ & $9.5 \pm 1.3^{\mathrm{a}}$ & $10.5 \pm 2.1^{\mathrm{a}}$ & $9.3 \pm 1.3^{\mathrm{a}}$ & $10.6 \pm 1.4^{\mathrm{a}}$ \\
\hline 5 & $9.7 \pm 1.3^{a}$ & $9.1 \pm 1.7^{\mathrm{a}}$ & $9.4 \pm 1.5^{a}$ & $9.1 \pm 1.5^{\mathrm{a}}$ & $9.5 \pm 1.3^{a}$ & $9.5 \pm 1.1^{\mathrm{a}}$ & $9.6 \pm 1.3^{a}$ & $9.7 \pm 1.1^{\mathrm{a}}$ \\
\hline 6 & $10.0 \pm 1.0^{\mathrm{a}}$ & $9.0 \pm 1.2^{\mathrm{a}}$ & $10.0 \pm 1.5^{\mathrm{a}}$ & $9.5 \pm 1.2^{\mathrm{a}}$ & $9.8 \pm 1.5^{\mathrm{a}}$ & $9.9 \pm 1.9^{a}$ & $9.9 \pm 1.7^{a}$ & $10.1 \pm 1.5^{\mathrm{a}}$ \\
\hline 7 & $9.9 \pm 1.0^{a}$ & $9.6 \pm 1.2^{\mathrm{a}}$ & $9.8 \pm 1.0^{a}$ & $9.5 \pm 1.5^{a}$ & $9.5 \pm 1.6^{a}$ & $9.7 \pm 1.7^{a}$ & $9.3 \pm 1.6^{\mathrm{a}}$ & $9.5 \pm 1.7^{a}$ \\
\hline 8 & $9.6 \pm 1.6^{a}$ & $9.8 \pm 1.4^{\mathrm{a}}$ & $9.5 \pm 1.5^{a}$ & $9.7 \pm 1.4^{a}$ & $9.7 \pm 1.8^{a}$ & $9.8 \pm 1.1^{\mathrm{a}}$ & $9.5 \pm 1.7^{a}$ & $9.8 \pm 1.7^{a}$ \\
\hline 9 & $9.8 \pm 1.7^{a}$ & $9.8 \pm 1.8^{\mathrm{a}}$ & $9.7 \pm 1.7^{a}$ & $9.9 \pm 1.5^{\mathrm{a}}$ & $10.2 \pm 1.8^{\mathrm{a}}$ & $10.0 \pm 1.1^{\mathrm{a}}$ & $10.0 \pm 1.2^{\mathrm{a}}$ & $10.5 \pm 1.2^{\mathrm{a}}$ \\
\hline 10 & $9.5 \pm 1.0^{\mathrm{a}}$ & $9.8 \pm 1.4^{\mathrm{a}}$ & $9.9 \pm 2.0^{\mathrm{a}}$ & $9.8 \pm 1.5^{\mathrm{a}}$ & $9.7 \pm 1.5^{\mathrm{a}}$ & $9.8 \pm 1.1^{\mathrm{a}}$ & $9.8 \pm 1.9^{\mathrm{a}}$ & $9.7 \pm 1.1^{\mathrm{a}}$ \\
\hline
\end{tabular}

$a, b, c$ Diferentes literales entre filas indican diferencia estadística ( $p<0.05 ;$ prueba de Tukey); T1= Amamantamiento restringido, destete precoz y complemento alimenticio; T2= Amamantamiento continuo sin complemento alimenticio.

\section{DISCUSIÓN}

En este estudio, se comparó el comportamiento productivo, bienestar físico y fisiológico de los becerros sometidos a dos estrategias de crianza. Las diferencias entre tratamientos, aquí encontradas, son atribuibles al proceso de adaptación en el consumo de alimento solido (cantidad y tiempo) y al desarrollo a nivel gastrointestinal necesario para la digestibilidad y absorción de nutrientes a partir de alimentos sólidos $(8,9)$ de los animales del tratamiento T1 comparado con los de T2. El proceso de adaptación en T1 inició a partir de que los becerros se separaron de las madres, a los 15 días de nacidos, hasta ser destetados a los 3.5 
meses de edad, a diferencia de los becerros del tratamiento T2 que permanecieron con sus madres hasta los 7 meses de edad, cuando fueron destetados. Este proceso de adaptación también fue observado por Lambertz et al (5) en becerros separados de las madres a las 4 horas de nacidos, a los cuales les fue suministrado leche ( 6 a 8 L/día) con una mamila hasta los 36 días de edad, y a partir de ese momento, la ingesta de leche disminuyó a 4 L/día hasta el destete ( 6 u 8 semanas). Cabe mencionar que además se les ofreció alimento peletizado para becerros ( $22 \%$ PC; EM= $2.63 \mathrm{Mcal} / \mathrm{kg}$ ), paja de avena ( $8 \% \mathrm{PC} ; \mathrm{EM}=1.55 \mathrm{Mcal} / \mathrm{kg})$ y agua ad libitum. En adición, en dicho estudio, se reporta que los becerros destetados a las 8 semanas comparados con los de 6 semanas, tuvieron mayor consumo de alimento sólido (1.36 y 0.40 $\mathrm{kg} /$ día) y ganancia de peso al destete (0.79 y $0.34 \mathrm{~kg} / \mathrm{día}$ ), respectivamente.

Esos autores, también mencionan, que el mismo comportamiento posdestete (150 días de edad) se observó en los becerros destetados a las 8 semanas con respecto a 6 semanas para el consumo de alimento ( 2.51 y $1.16 \mathrm{~kg} /$ día) y ganancia de peso al destete (1.05 y $0.35 \mathrm{~kg} /$ día). Ellos atribuyeron esas diferencias al tiempo de exposición de los animales de 8 semanas a la ingesta de alimento sólido y mejor desarrollo gastrointestinal comparado con los de 6 semanas. En el presente experimento, aunque los cambios en peso corporal fueron mejores en el tratamiento T2 comparados con T1 durante la etapa de destete, la tendencia en T1 fue hacia ganar peso, lo cual indicó que existió una disminución en la dependencia de leche materna y una rápida adaptación al consumo de alimento sólido $(5,9,18)$. de-Castro et al (19) reportaron que, la ganancia diaria de peso (1.12 kg/día) fue más eficiente al dar alimento a los becerros por periodos más prolongados ( $\geq 180$ días) en comparación con periodos cortos ( $\leq 90$ días; 0.91 $\mathrm{kg} /$ día), además de ser más costoso. De ahí el beneficio de dar complementación alimenticia a becerros durante la etapa posdestete.

Los cambios de peso corporal posdestete fueron mejores en los becerros del tratamiento T1 con respecto a los del tratamiento $\mathrm{T} 2$, observándose una tendencia negativa en la ganancia diaria de peso y en la ganancia de peso total en T2. Lo cual fue consecuencia de que los becerros del T2 estaban en la etapa de adaptación (destete) que los becerros del T1 ya habían pasado. Lo relevante en los cambios de peso corporal, fue el comportamiento que tuvieron los animales de
T1 con respecto a los de T2; donde los primeros no presentaron pérdida evidente de peso en la etapa de la separación (15 días de edad), destete o posdestete, comparado con los del T2, los cuales después del destete perdieron peso (Figura 1). Esto se atribuyó a la dependencia de leche materna y limitada capacidad de comer alimentos sólidos $(20,21)$. Esa circunstancia mermo el peso ganado hasta ese momento con el amamantamiento continuo, y que finalmente los puso en desventaja con los becerros del tratamiento T1. Esto se explica, porque a partir del deteste de los animales del grupo T2 (7 meses de edad) y hasta los 10 meses de edad, el comportamiento en los cambios de peso de los becerros del tratamiento T1 mejoró y el de los de T2 disminuyó.

Pérez et al (13) reportaron que animales con destete temporal (24, 48 o 72 horas) a los 25 o 45 días de nacidos, y destetados a los 150 días de edad $(130 \mathrm{~kg})$, tuvieron mayor ganancia de peso al destete $(200 \mathrm{~kg}$ ) y posdestete (270 días de edad) cuando permanecieron más tiempo aislados de la madre 48 o 72 horas. Esto fue independientemente de la edad, comparado con los animales del grupo control, y con aquellos destetados por 24 horas que pesaron $175 \mathrm{~kg}$. Estas diferencias fueron atribuidas al proceso previo de separación que habían experimentado los animales, lo cual preparó a los becerros separados por 48 o 72 horas, para estar mejor adaptados a no tener a la madre presente, y los forzó a comer más alimento sólido.

Pazoki (22) y Mageste et al (23) mencionan que la complementación alimenticia antes y después del destete, mejora la ganancia diaria de peso ( $\geq 0.800 \mathrm{~kg} /$ día) y el desarrollo físico de los animales. Sin embargo, en posdestete, los animales con exposición previa al consumo de alimento sólido (alimento concentrado y/o pasto) desarrollaron mejores condiciones físicas y químicas que permitieron al rumiante una mejor estabilidad del pH ruminal, así como, destinar más tiempo a la rumia de los alimentos (aumento de la musculatura ruminal). En adición, se menciona que se estimula y mejoran los atributos morfométricos de la pared ruminal a nivel micro y macroscópico, que aumentan y mejoran la digestibilidad de nutrientes y el estatus metabólico; características que aportan mejoras productivas a los becerros y económicas a los productores.

Desde el punto de vista económico y productivo, es mejor un becerro que gana peso, a uno que 
pierde peso (18). El disminuir la dependencia a la leche y aumentar la ingesta de alimentos sólidos, permite destetar becerros machos a corta edad ( $\leq 5$ meses de vida) con buen peso ( $\geq 209 \mathrm{~kg}$ ), para ser enviados a la engorda y finalizados a los 10.5 meses de edad, y con un peso de $451 \mathrm{~kg}$ (18). Así mismo, se estimula que las hembras pueden iniciar su vida reproductiva a $\leq 16$ meses de edad (23), ambas circunstancias económicamente rentables. Por lo tanto, el amamantamiento controlado, destete precoz, temporal o cualquier otra técnica zootécnica que emplee la separación temporal o permanente del becerro de la madre, aunado al estímulo de manera paulatina del consumo de alimentos sólidos (alimento balanceado y/o forraje), es una herramienta eficaz que disminuye la dependencia a la leche materna. En adición, inducir un destete temprano, incrementa la ganancia de peso y el desarrollo físico y productivo de los animales en corto plazo, de manera económicamente rentable.

La separación temporal o permanente del becerro, con fines de destete o mejora productiva, lo afecta debido a la pérdida o ausencia de la madre, el acceso a la ubre para consumir leche, así como debido a cambios en la dieta, en la estructura social y entorno físico, que alteran su bienestar físico, fisiológico y de comportamiento $(14,24)$. Para determinar el efecto de esos cambios, se han propuesto indicadores que miden el estado de bienestar animal, reflejado directamente en el estrés que experimenta el becerro durante su proceso productivo, y que alteran la concentración plasmática de cortisol, el ritmo cardiaco, la temperatura corporal y locomoción $(2,10,24)$. Sin embargo, los cambios en la concentración de cortisol parecen ser particularmente útiles como un indicador de estrés agudo, así como del funcionamiento del sistema hipotalámico-pituitariaadrenocortical, en respuesta a experiencias emocionales y físicas $(2,10)$.

Las diferencias en la concentración plasmática de cortisol, analizadas considerando el promedio de las dos muestras de cortisol, fueron evidentes el día 0 ( $(T 1 ; 32.8$ y T2; 10.52; $\mathrm{p}<0.05)$. Sin embargo, el día 1 ( $\mathrm{T} 1 ; 26.2$ y T2; 11.4), 2 (T1; 19.9 y T2; 10.1) y 3 (T1; 15.3 y T2; 10.7), aunque se encontró diferencia significativa, la concentración disminuyó conforme pasaron los días y, para el día 4 ( $T 1 ; 9.8$ y T2; 9.9), los becerros del tratamiento T1 y T2 tuvieron similar concentración plasmática ( $p>0.05$; Tabla 2). Por lo tanto, la separación del becerro por el amamantamiento controlado afecta los niveles de estrés del animal, pero, invariablemente ese estado fisiológico se estabiliza rápidamente, indicando que los animales se adaptan a la separación en pocos días.

Acevedo et al (10), reportan que las concentraciones de cortisol plasmático entre becerros con amamantamiento restringido (separación temporal, permitiendo mamar una vez al día por 30 min de 8:00 am a 8:30 am y consumo de forraje), y destete temporal (DT; separación permanente, sin ningún tipo de contacto por 72 horas, con consumo de forraje), fueron mayores en el destete temporal $(23.36 \mathrm{ng} / \mathrm{mL}), 24$ horas después de iniciado el tratamiento. Sin embargo, estos valores decrecieron significativamente a niveles similares a los becerros con amamantamiento restringido al segundo día (48 horas) (DT; 10.99 y AR; $11.44 \mathrm{ng} / \mathrm{mL}$ ), y el tercer día (72 horas) no se encontraron diferencias entre los tratamientos $(p>0.05)$. Esto se atribuyó a que los animales con amamantamiento restringido redujeron el estrés mediante el contacto físico que se les provee de manera controlada y temporal (cuando se les permite mamar) comparado con los becerros sometidos a destete temporal que, fueron separados totalmente de la madre. Aunque, al final los animales con destete temporal al segundo día del experimento ya se habían acostumbrado al aislamiento.

Por otro lado, Pérez-Torres et al (2) determinaron el efecto del estrés causado por el destete temporal según la edad de los animales ( 25 y 45 días de nacidos), a los que, separaron de manera permanente de las madres por 24, 48 ○ 72 horas, proporcionándoseles alimento comercial ( $16 \%$ de PC), sal mineral y agua ad libitum. Se observó, que los becerros de 25 días de vida tuvieron mayor concentración de cortisol plasmático $(\mathrm{ng} / \mathrm{mL})(10.3,5.3$ y 2.0 , a las 24 , 48 y 72 horas, respectivamente) comparados con los de 45 días $(6.2,2.1$ y 0.8 , a las 24,48 y 72 horas, respectivamente). Estos resultados se atribuyeron al vínculo vaca-becerro y a una mayor dependencia social de los animales jóvenes comparados con los de edad mayor, aunque la concentración plasmática de cortisol decreció marcadamente en ambos grupos a las 72 horas después de la separación.

Por lo tanto, considerando los resultados de estos estudios y él presente, se puede inferir que la separación de los becerros en cualquiera de sus modalidades de destete temporal o amamantamiento restringido genera estrés en los animales. Sin embargo, esta condición 
fisiológica es una respuesta y/o reacción natural de los animales al cambio de sus circunstancias, la cual disminuirá hasta normalizarse en un periodo promedio de 48 a 96 horas posteriores a la separación. Finalmente, el proceso de destete es mejor, estimulando en los becerros el consumo de alimento sólido, de manera paulatina y disminuyendo su dependencia a la leche materna, de manera que puedan adaptarse, tranquilamente y sin efectos negativos en la ganancia de peso, salud y desarrollo físico.
En conclusión, el amamantamiento restringido, destete precoz y la complementación alimenticia permitieron el destete de los becerros machos y hembras a los 3.5 meses de vida e incrementaron la ganancia de peso posdestete. El peso vivo y ganancia de peso posdestete fue mejor en los animales del tratamiento $\mathrm{T} 1$. Los niveles de cortisol plasmáticos incrementaron al inicio del estudio, pero disminuyeron posteriormente.

\section{Conflicto de interés}

Los autores de este articulo declaran no tener conflictos de interés.

\section{REFERENCIAS}

1. Enríquez $D H$, Ungerfeld $R$, Quintans $G$, Guidoni AL, Hötzel MJ. The effects of alternative weaning methods on behaviour in beef calves. Livest Sci. 2010; 128(13) : 20-27. https://doi.org/10.1016/j. livsci.2009.10.007

2. Pérez-Torres $L$, Orihuela $A$, Corro $M$, Rubio I, Alonso MA, Galina CS. Effects of separation time on behavioral and physiological characteristics of Brahman cows and their calves. Appl Anim Behav Sci. 2016; 179:17-22. https://doi.org/10.1016/j. applanim.2016.03.010

3. Arthington JD, Spears JW, Miller DC. The effect of early weaning on feedlot performance and measurements of stress in beef calves. J Anim Sci. 2005; 83(4):933-939. https:// doi.org/10.2527/2005.834933x

4. Odhiambo JF, Rhinehart JD, Helmondollar R, Pritchard JY, Osborne PI, Felton EE et al. Effect of weaning regimen on energy profile and reproductive performance of beef cows. J Anim Sci. 2009; 87(7):2428-2436. https://doi.org/10.2527/jas.2008-1138

5. Lambertz Ch, Farke-Röver A, Gauly M. Effects of sex and age on behavior and weight gain in beef calves after abrupt weaning. Anim Sci J. 2015; 86(3):345-350. https://doi.org/10.1111/asj.12285
6 Martins PGMA, Arthington JD, Cooke RF, Lamb CG, Araujo DB, Torres CAA, Guimaraes, JD, Mancio AB. Evaluation of beef cow and calf separation systems to improve reproductive performance of first-calf cows. Livest Sci. 2012; 150(1-3):74-79. https:// doi.org/10.1016/j.livsci.2012.08.003

7. Waterman RC, Geary TW, Paterson JA, Lipsey RJ. Early weaning in Northern Great Plains beef cattle production systems: I. Performance and reproductive response in range beef cows. Livest Sci. 2012; 148(1-2):26-35. https://doi.org/10.1016/j. livsci.2012.05.004

8. Eckert E, Brown HE, Leslie KE, DeVries TJ, Steele MA. Weaning age affects growth, feed intake, gastrointestinal development, and behavior in Holstein calves fed an elevated plane of nutrition during the preweaning stage. J Dairy Sci. 2015; 98(9):6315-6326. https://doi.org/10.3168/jds.2014-9062

9. Mageste de Almeida D, Marcondes MI, Navajas-Rennó L, Soares-Martins L, Contreras-Marquez DE, Castaño-Villadiego $F$, et al. Supplementation strategies for Nellore female calves in creep feeding to improve the performance: nutritional and metabolic responses. Trop Anim Health Prod. 2018; 50(8):1778-1785. https://doi. org/10.1007/s11250-018-1619-2 
10. Acevedo N, Hernández C, Orihuela A, Lidfors LM, Berg C. Effect of restricted suckling or temporal weaning on some physiological and behavioural stress parameters in Zebu cattle (Bos indicus). J Anim Sci. 2005; 18(8):1176-1181. https://doi.org/10.5713/ ajas. 2005.1176

11. Galina CS, Rubio I, Basurto $H$, Orihuela A. Consequences of different suckling systems for reproductive activity and productivity of cattle in tropical conditions. Appl Anim Behav Sci. 2001; 72(3):255-262. https:// doi.org/10.1016/S0168-1591(01)00115-0

12. Mejía-Bautista GT, Magaña JG, SeguraCorrea JC, Delgado R, Estrada-León RJ. Comportamiento reproductivo y productivo de vacas Bos indicus, Bos taurus y sus cruces en un sistema de producción vaca: cría en Yucatán, México. Trop Subtrop Agroecosyst. 2010; 12(2):289-301. http:// www.revista.ccba.uady.mx/ojs/index.php/ TSA/article/view/403/375

13. Pérez LI, Orihuela $A$, Galina CS, Rubio $I$, Corro M, Cohen A, et al. Effect of different periods of maternal deprivation on behavioral and cortisol responses at weaning and subsequent growth rate in zebu (Bos indicus) type cattle. Livest Sci. 2017; 197:17-21. https://doi.org/10.1016/j. $\underline{\text { livsci.2016.12.006 }}$

14. Ungerfeld R, Quintans G, Hötze MJ. Minimizing cows' stress when calves were early weaned using the two-step method with nose flaps. Animal. 2016; 10(11):1871-1876. https:// doi.org/10.1017/S1751731116000793

15. NOM-062-ZOO-1999. Norma oficial mexicana. Especificaciones técnicas para la producción, cuidado y uso de los animales de laboratorio. Diario Oficial de la Federación. 1999. https://www.gob.mx/ cms/uploads/attachment/file/203498/NOM062-ZOO-1999 220801.pdf

16. Instituto Nacional de Estadística y Geografía (INEGI). Ubicación geográfica, condiciones climáticas y orográficas. INEGI: México; 2018. https://www.inegi.org.mx/app/mapa/ espacioydatos/default.aspx?ag $=270120001$

17. SPSS Inc. Released 2010. IBM-SPSS Statistics for Windows, Version 19.0. Chicago: SPSS Inc.https://www.ibm.com/ analytics/spss-statistics-software
18. Blanco M, Villalba D, Ripoll G, Sauerwein $\mathrm{H}$, Casasus I. 2008. Effects of pre-weaning concentrate feeding on calf performance, carcass and meat quality of autumn-born bull calves weaned at 90 or 150 days of age. Animal. 2(5):779-789. https://doi. org/10.1017/S1751731108001808

19. de-Castro-de-Menezes GC, ValadaresFilho S, Lopez-Villalobos N, Mendes-Ruas JR, Detmann E, Zanetti D, et al. Effect of feeding strategies on weaning weight and milk production of Holstein $\times$ Zebu calves in dual purpose milk production systems. Trop Anim Health Prod. 2015; 47(6):1095-1100. https://doi.org/10.1007/s11250-015-0832-5

20. Khan MA, Weary DM, von-Keyserlingk MAG. Hay intake improves performance and rumen development of calves fed higher quantities of milk. J Dairy Sci. 2011; 94(7):3547-3553. https://doi.org/10.3168/ jds.2010-3871

21. Soares-Martins L, Fonseca-Paulino $M$, Navajas-Rennó L, Detmann E, Mageste de Almeida D, Maza-Ortega R, et al. Creep feeding effects on male Nellore calves influencing behavior and performance of their dams. Trop Anim Health Prod. 2017; 49(8):1669-1676. https://doi.org/10.1007/ s11250-017-1375-8

22. Pazoki A, Ghorbania GR, Kargarb S, Sadeghi-Sefidmazgia A, Drackleyc J K, Ghaffari MH. Growth performance, nutrient digestibility, ruminal fermentation, and rumen development of calves during transition from liquid to solid feed: Effects of physical form of starter feed and forage provision. Anim Feed Technol. 2017; 234:173-185. https://doi.org/10.1016/j. anifeedsci.2017.06.004

23. Mageste de Almeida D, Marcondes MI, Navajas-Rennó L, Pereira-Silva LH, SoaresMartins L, Contreras-Marquez DE, et al. Nutritional planning for Nellore heifers postweaning to conception at 15 months of age: performance and nutritional, metabolic, and reproductive responses. Trop Anim Health Prod. 2018; 51(1):79-87. https:// doi.org/10.1007/s11250-018-1662-z

24. Enríquez D, Hötzel MJ, Ungerfeld R. Minimising the stress of weaning of beef calves: a review. Acta Vet Scand. 2011; 53(28):2-8. https://doi.org/10.1186/1751$\underline{0147-53-28}$ 